\title{
$1 \quad$ Authentication of paprika using HPLC-UV fingerprints
}

2

$$
\text { Xavier Cetóa }{ }^{\mathrm{a}} \text {, Cristina Sánchez }{ }^{\mathrm{a}} \text {, Núria Serrano }{ }^{\mathrm{a}} \text {, José Manuel Díaz Cruz }{ }^{\mathrm{a}, *} \text {, Oscar }
$$

Núñez ${ }^{\text {a,b,c }}$

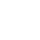

${ }^{a}$ Department of Chemical Engineering and Analytical Chemistry, Faculty of Chemistry, Universitat de Barcelona, 08028 Barcelona, Spain

${ }^{b}$ Research Institute in Food Nutrition and Food Safety, University of Barcelona, 08901, (1) Santa Coloma de Gramanet, Spain

${ }^{c}$ Serra Hunter Fellow, Generalitat de Catalunya, Barcelona, Spain

*to whom correspondence should be addressed, e-mail: josemanuel.diaz@ub.edu

\section{Abstract}

In this work we combine simple extraction and HPLC-UV methodologies with chemometric pattern-recognition strategies in order to obtain characteristic fingerprints of phenolic compounds that allow the authentication of paprika samples. To illustrate the potential of the proposed approach, two different adulteration scenarios were considered, namely adulteration of paprika based on its type (sweet, bittersweet and spicy) as well as on its region (Murcia, la Vera and Czech Republic). Upon preparation of a proper set of samples, they were analysed using a $\mathrm{C}_{18}$ reversed-phase column and registered chromatograms were then compressed employing fast Fourier transform (FFT) to reduce the large dimensionality of the data set, while preserving all relevant features. Next, data were analysed using linear discriminant analysis (LDA) for the 
25 qualitative discrimination of adulterated samples, followed by partial least-squares

26 regression (PLS) modelling to quantitatively assess the adulteration degree.

27

28 Keywords: paprika; food authentication; adulteration; liquid chromatography; partial

29 least-squares regression 


\section{Introduction}

In an effort to promote and protect the quality of regional foods, geographical indications (e.g. protected designation of origin, PDO), regulatory boards assess that producers comply with the specific technical production conditions and performs regular controls (including sensory and analytical examinations, as well as stock statements and verification of movements) (Dias \& Mendes, 2018). However, despite these controls, there is a demand of new analytical low-cost methods needed to assess both authenticity and fulfilment of quality standards (Danezis, Tsagkaris, Camin, Brusic, \& Georgiou, 2016; Galvin-King, Haughey, \& Elliott, 2018). Particularly, this is critical when trying to assess the authenticity of local natural foods. Unfortunately, there is a lack of methods able to classify food samples, since usually there is not any specific compound directly related to food origin or quality that could be determined using conventional analytical techniques.

A system capable to perform such task should simultaneously detect a large spectrum of compounds and provide comprehensive information of the sample. In this regards, current approaches for quality control are shifting from compound-oriented to pattern-oriented strategies (Cavanna, Righetti, Elliott, \& Suman, 2018; CuadrosRodríguez, Ruiz-Samblás, Valverde-Som, Pérez-Castaño, \& González-Casado, 2016; Esteki, Shahsavari, \& Simal-Gandara, 2019; Zeng et al., 2008). This means developing methodologies for the simultaneous detection of many compounds and the further pattern recognition analysis of the data, instead of focusing on the quantification of a few specific substances. The main advantage of pattern-oriented approaches is that they do not require any prior knowledge of the sample composition in order to succeed, but even more, they can be used to assess those key (bio)markers. 
In recent years, there has been an increased interest and knowledge on the presence of bioactive compounds in food, as well as on the role of such substances on the quality and health benefits of food products, which has to be guaranteed (Johanningsmeier, Harris, \& Klevorn, 2016; Kris-Etherton et al., 2002). Bioactive compounds distribution in most natural foods can be related to the specific products varieties, processing technologies, production regions and climate conditions (Baenas, Belović, Ilic, Moreno,

60 \& García-Viguera, 2019; Mudrić et al., 2017). Most of these compounds are powerful antioxidants needed for the functioning of plant cells, with huge health benefits upon its ingestion as they can act as free radical scavengers and inhibitors of lipoprotein oxidation, providing a protective effect against aging pathologies like cardiovascular diseases or cancers mutation (Kim et al., 2016; Quideau, Deffieux, Douat-Casassus, \& Pouységu, 2011).

Paprika, sometimes also referred to as chilli pepper, is a characteristic red seasoning powder obtained from the drying and grinding of certain varieties of red peppers (Capsicum annuum L.) (Pérez-Gálvez, et al., 2005). There are three important varieties of paprika: sweet, bittersweet, and spicy. The two most known varieties of paprika in Spain, and the only ones with a PDO, come from the region of "la Vera" in Cáceres (Extremadura) and from "Murcia" (Commission Regulation (EEC), 11 February 2000, 24 November 2006). Among the different bioactive substances found in paprika, phenolic compounds are especially important, and their distribution may be related to the different red pepper varieties (Baenas et al., 2019; Mudrić et al., 2017; Quideau et al., 2011).

In this context, herein we investigate on the capabilities of combining liquid chromatography, to obtain a profile of the phenolic content of paprika's, with 
squares regression (PLS) for the extraction of a characteristic fingerprint that allow the

80 authentication of paprika samples.

\section{Experimental}

\subsection{Reagents and materials}

Methanol (UHPLC-gradient grade), formic acid 98\%, acetonitrile, absolute ethanol and acetone were purchased from Panreac (Barcelona, Spain). Standards of phenolic compounds were obtained from Sigma-Aldrich (St. Louis, MO, USA), from which stock solutions of $1000 \mathrm{mg} / \mathrm{L}$ were prepared in methanol and stored in amber glass vials. Deionized water was obtained from a Milli-Q system (Millipore, Billerica, MA, USA).

\subsection{Paprika samples}

Authentication of paprika was studied from two different points of view. On the one side, adulteration with paprika from other regions was considered, whereas on the other side, adulteration with paprika from other varieties was also evaluated.

To this aim, paprika samples from three different regions (La Vera, Murcia and

Czech Republic) were considered. Among the samples of every region, there were

97 different types of paprika (sweet, bittersweet and spicy in the samples from La Vera, and sweet and spicy in the samples from Murcia and Czech Republic). The samples were purchased directly from producers or from different local shops. Adulteration of

100 paprika was made in two ways. In the study about paprika types, 24 mixtures were

101 prepared with different proportions of the varieties sweet, bittersweet and spicy of 102 paprika from La Vera. For each variety, 12 different proportions were considered (0, $0.01,0.02,0.1,0.2,0.4,0.6,0.8,0.9,0.98,0.99$ and 1$)$, according to the design of 24 
104 experiments summarised in Table 1. Every mixture was prepared twice, which means

10548 samples to be analysed. In the study about paprika regions, 24 mixtures were 106 prepared with samples of the type spicy from the regions of La Vera, Murcia and Czech

107 Republic. As in the previous study, the experimental design of Table 1 was used and the 108 samples were prepared twice, also producing a total of 48 samples.

109 Prior to its analysis, paprika samples were subjected to a extraction stage by 110 sonication and centrifugation in water:acetonitrile (20:80 v/v) (Cetó et al., 2018).

111 Briefly, $0.3 \mathrm{~g}$ of paprika were weighted, dispersed in $3 \mathrm{~mL}$ of solution and vortexed for

$1121 \mathrm{~min}$. Next, samples were sonicated for $15 \mathrm{~min}$ and centrifuged at $4500 \mathrm{rpm}$ for 30 113 min. Finally, the supernatant was filtered through $0.45 \mu \mathrm{m}$ nylon filters, and samples 114 stored at $4{ }^{\circ} \mathrm{C}$ until their analysis.

\subsection{Chromatographic analysis}

117 HPLC analysis was carried out in an Agilent 1200 Series instrument (Palo Alto, CA,

118 USA) equipped with a G1311A quaternary pump, a G1322A vacuum degasser, a 119 G1329A autosampler and a G1314B ultraviolet-visible detector; all of them controlled 120 with the Agilent ChemStation software package.

121 Chromatographic fingerprints were obtained with a reverse phase Kinetex $\mathrm{C}_{18}$ column $(2.6 \mu \mathrm{m} \mathrm{C18} 100 \AA$, 100 x $4.6 \mathrm{~mm})$ from Phenomenex (Torrance, CA, USA) at

123 room temperature. For the elution, a mixture of Milli-Q water containing $0.1 \%$ formic

124 acid (solvent A) and methanol (solvent B) were used as the mobile phase components at 125 a flow rate of $1 \mathrm{~mL} / \mathrm{min}$, and with the following gradient: $0-2 \mathrm{~min}$, isocratic step at $5 \%$ $126 \mathrm{~B} ; 2-4$ min linear gradient from 5 to $25 \% \mathrm{~B} ; 4-12 \mathrm{~min}$, at $25 \% \mathrm{~B} ; 12-14 \mathrm{~min}$, from 25 to $12745 \% \mathrm{~B} ; 14-16 \mathrm{~min}$, at $45 \% \mathrm{~B} ; 16-18 \mathrm{~min}$, from 45 to $95 \% \mathrm{~B} ; 18-20 \mathrm{~min}$, at $95 \% \mathrm{~B} ; 20-$ $12821 \mathrm{~min}$, back to initial conditions at $5 \% \mathrm{~B}$; and from $21-30 \mathrm{~min}$, at $5 \% \mathrm{~B}$ for column re- 
129 equilibration (Cetó et al., 2018). Injection volume was $20 \mu \mathrm{L}$, and UV absorption

130 registered at $280 \mathrm{~nm}$ every $291 \mathrm{~ms}$.

131 Every paprika sample was injected by triplicate, which generated 144

132 chromatograms in the study about paprika types and 144 chromatograms more in the

133 study about paprika regions.

\subsection{Chemometric analysis}

136 The resulting chromatograms were first baseline corrected by polynomial fitting and

137 subtraction of the background and compressed using fast Fourier transform (FFT), and

138 then submitted to linear discriminant analysis (LDA) and partial least squares regression

139 (PLS) by means of home-made programs implemented in Matlab 7.1 (MathWorks,

140 Natick, MA, USA) (Cetó, Céspedes, \& del Valle, 2013).

141 Briefly, FFT was used to reduce the large dimensionality of the recorded data, while

142 LDA was used to actually attempt its categorization based on the adulteration degree.

143 Finally, in order to numerically quantify the degree of adulteration, PLS was employed.

144 In both cases, the set of samples was randomly split between two subsets: training and

145 testing, in the ratio 2:1 to ensure unbiased results were obtained from the models.

\section{3. Results and Discussion}

148 As discussed earlier, it is very complicated to achieve the authentication of food

149 samples from the concentration profiles of specific compounds obtained from their

150 targeted analysis. Oppositely, completely non-targeted analysis has also the drawback

151 that much more features or descriptors will be required (including many that will turn to

152 be non-relevant), thus hindering the data processing stage as well as possibly demoting 153 model performance. 
154 In this direction, we have developed and applied a chromatographic method for the

155 profiling of phenolic compounds present in paprika (Figure 1), and we hypothesize on

156 the potential of this method in combination with chemometric analysis for the

157 authentication of paprika samples. To illustrate its potential, two different scenarios

158 were explored taking into account paprika classification, namely the adulteration with

159 different varieties and the adulteration with paprika from different regions (PDO's).

160 Moreover, not only the qualitative authentication was considered, but also the 161 quantification of the adulteration degree was attempted. The results obtained are 162 presented over the next sections.

\subsection{Authentication of paprika based on its type}

165 The first study case was to attempt the authentication of adulterated sweet, bittersweet and spicy paprika samples from La Vera according to the levels reported in

167 Table 1. Upon preparation of the set of adulterated samples, they were subjected to the 168 extraction procedure and the chromatographic analysis described above, which 169 produced a set of 144 chromatograms with characteristic fingerprints as these shown in $170 \quad$ Figure 1.

171 Upon measurement of all the set of samples, the next step was to attempt its 172 discrimination with the aid of chemometric methods. However, given the large 173 dimensionality of the recorded data, chromatograms were first compressed down to 512 174 coefficients with the aid of FFT algorithm. This allowed a reduction of over $95.8 \%$ on 175 the pattern matrix without any loss of significant information and also a notorious 176 decrease in the instrumental noise (Cetó et al., 2013).

177 The chosen pattern recognition method to attempt the discrimination of the 178 adulterated samples was LDA, taking the different adulteration levels (i.e, the 24 
179 mixture proportions) as the classes into which the samples were divided and the

180 calculated Fourier coefficients as the pattern matrix. To further remove non-relevant 181 variables with lower or none relevance to the classification task, a stepwise inclusion 182 method was used so as to select the minimum set of coefficients to perform the 183 prediction task with the optimum performance (Johnson \& Wichein, 2007).

184 The two dimensional score plot obtained after LDA is shown in Figure 2. Despite its 185 complexity given the large number of classes considered (24), there are some interesting 186 trends that can be observed. Firstly, it is important to note how the three classes 187 corresponding to the pure (non-adulterated) paprika samples are the ones taking the 188 extreme values for both discriminating functions (DFs), or in other words, appear at the 189 extremes of the plot. That is, $\mathrm{C} 1$ corresponding to sweet samples in the right bottom of 190 the plot, opposite to it there is C17 corresponding to spicy samples and on the top in 191 between those two there is C9 corresponding to bittersweet samples. More interestingly, 192 it can also be observed how two big clusters appear distinguishing spicy adulterated 193 samples from sweet and bittersweet adulterated ones. That is, if we imagine a line going 194 from the top left to the bottom right, we can see how those clusters would be separated 195 by it. Even more, we can notice how intra-clusters distance is bigger for this subset of 196 samples compared to the other, thus indicating that adulteration of spicy paprika 197 samples with other types of paprika is much more noticeable. This fact might be due to 198 the much higher concentrations of capsaicinoids in spicy paprika in comparison to the 199 other two types, which leads to a significant decrease of its concentration in the 200 mixtures. Lastly, despite the apparent overlapping that might be seen with some classes 201 in this 2D representation (e.g. C18/C20), this is not an issue as when also DF3 is 202 plotted (which represents $9.48 \%$ of the total model variance), it can be seen how the 203 clusters are clearly discriminated (with respective centroids coordinates of ca. 15 and -5, 
respectively). In this regard, it has to be kept on mind that the actual model has a total of

20523 DFs, which are the ones used to numerically assign the samples to each of the 206 classes.

207 Next, in order to numerically assess the performance of the model, confusion 208 matrixes were built (data not shown). Classification rate for the training and testing 209 subsets was $100 \%$ and $81.3 \%$, respectively; the latter being slightly lower due to some 210 miss-classification between sweet and bittersweet adulterated samples at the lower 211 considered levels. Moreover, performance of the model was also evaluated in terms of 212 sensitivity, specificity and precision values (averaged for the classes) (Cetó, Voelcker, 213 \& Prieto-Simón, 2016), achieving a 81.3\%, 99.2\% and 85.4\%, respectively.

214 Upon confirmation of the capability of the method to discriminate adulterated 215 samples, and even more, to actually discriminate between different levels of 216 adulteration, the next step was to attempt to numerically predict the actual degree of 217 adulteration. To this aim, PLS was used instead of LDA as the modelling tool, using as 218 before the calculated Fourier coefficients as the pattern matrix, but taking the actual 219 percentage of adulteration rather than the classes as the target matrix.

220 As an example, the comparison graph of the predicted vs. expected percentage of adulteration for the mixtures of sweet and bittersweet samples is shown in Figure 3 . As can be seen, a good trend is obtained, with fitted regression lines for both training and testing subsets almost indistinguishable from the ideal comparison line $(\mathrm{y}=\mathrm{x})$. That is,

224 with slope and correlation coefficients close to 1 , and intercept value close to 0 ; being 225 the theoretical values included in the $95 \%$ confidence interval. In this way, confirming 226 the potential of the approach not to only qualitatively discriminate between pure and 227 paprika adulterated with other varieties, but also to numerically quantify the degree of 228 adulteration. 


\subsection{Authentication of paprika based on its region}

To further assess the suitability of the proposed method for the authentication of paprika, not only adulteration within different varieties was considered, but also the potential fraud of not respecting the PDOs. That is, mixing paprika produced in different regions. As done with the previous scenario, the same experimental design was employed, considering spicy samples from three different regions: Murcia, la Vera and Czech Republic.

As before, upon measurement of all samples, the set of 144 chromatograms was compressed employing FFT and a qualitative LDA model was built to attempt its discrimination. The resulting scores plot is shown in Figure 4. In this case a very clear trend was observed-in the score plot, with the different classes taking almost a perfect triangular shape where each vertex corresponds to the unadulterated paprika samples and the mixed samples are distributed along the faces of the triangle, being sorted according to the degree of adulteration. That is, la Vera samples (C1) is in the left top of

244 the plot and opposite to Murcia samples (C9), which appear on the right sharing similar scores for DF2; meanwhile Czech Republic samples appear on the bottom, in between those two, with clear different values for DF2 evidencing that that coordinate basically discriminates Spanish and Czech samples. Very significant is also the increase in the percentage of accumulated variance only with the first two DFs, as in this case, the value goes up to ca. $91.6 \%$. A huge value that helps to explain why such a clear trend 250 has been obtained in the scores plot.

In order to numerically assess such a promising output, a confusion matrix was built (data not shown), from which the classification rate for the training and testing subsets was estimated as $100 \%$ and $95.8 \%$, respectively. Performance of the model was also 
254 evaluated in terms of sensitivity, specificity and precision values (averaged for the 255 classes) (Cetó et al., 2016), achieving a 95.8\%, 99.8\% and 97.2\%, respectively. All 256 these metrics confirm what could be somehow expected from the LDA score plot, i.e.

257 the fact that a clear identification is obtained for the authentication of paprika's region.

258 In this direction, we suspect that the superior performance observed in this case as 259 compared to the previous one might be due to the higher impact on the phenolic profile 260 derived from the different geographical climate conditions, but also to the fact that 261 different varieties might be cultivated over different areas, which exalts further this 262 different profiling (Mudrić et al., 2017).

263 Finally, a PLS model was also built to confirm what seems to be very clear from the

264 LDA scores plot in this case, and is the fact that the proposed chromatographic 265 approach has huge potential to be used to numerically predict the degree of adulteration.

266 As an example, the comparison graph of the predicted vs. expected percentage of

267 adulteration for the mixtures of la Vera and Murcia samples is shown in Figure 5. A 268 very good trend is obtained, with fitted regression lines for both training and testing 269 subsets almost indistinguishable from the ideal comparison line $(y=x)$, containing the 270 theoretical values of slope (1) and intercept (0) in the 95\% confidence interval. Thus, 271 the proposed methodology allows both the qualitative identification and the quantitative 272 determination of the degree of adulteration of paprika with paprika from other regions.

\section{Conclusions} methods in combination with chemometric analysis for the authentication of paprika samples. More specifically, we can confirm the hypothesis that the broad phenolic profile of paprika is significant enough to allow the discrimination of paprika samples 
279 given that phenolic distribution and content in natural food products seems to be related

280 to food features such as the plant/fruit/seed variety, the geographical climate conditions

281 of their production area, and the cultivation and manufacturing practices, among others.

282 Consequently, they could be a rich source of analytical information to carry out the

283 characterization, classification and authentication of food products as well as to detect

284 possible adulterations.

285 Overall, this work aims to demonstrate the advantages derived from the use of 286 chemometric methods as an alternative to specific-compound targeted classical analysis.

287 In this way, a biomimetic approach generates an overall fingerprint of the food products 288 analysed which allows to overcome the lack of knowledge of the compounds 289 responsible for certain characteristics and/or perceptions.

290

\section{Acknowledgments}

292 The authors acknowledge the support of Generalitat de Catalunya through the 293 projects 2017SGR-311 and 2017SGR-310. X. Cetó thanks the support of the Beatriu de 294 Pinós postdoctoral programme of the Government of Catalonia's Secretariat for 295 Universities and Research of the Ministry of Economy and Knowledge. 


\section{References}

298 Baenas, N., Belović, M., Ilic, N., Moreno, D. A., \& García-Viguera, C. (2019). Industrial use of pepper (Capsicum annum L.) derived products: Technological benefits and biological advantages. Food Chemistry, 274, 872-885. doi: https://doi.org/10.1016/j.foodchem.2018.09.047

Cavanna, D., Righetti, L., Elliott, C., \& Suman, M. (2018). The scientific challenges in moving from targeted to non-targeted mass spectrometric methods for food fraud analysis: A proposed validation workflow to bring about a harmonized approach. Trends in Food Science \& Technology, 80, 223-241. doi: https://doi.org/10.1016/j.tifs.2018.08.007

Cetó, X., Céspedes, F., \& del Valle, M. (2013). Comparison of methods for the processing of voltammetric electronic tongues data. Microchimica Acta, 180(5-6), 319-330. doi: 10.1007/s00604-012-0938-7

Cetó, X., Serrano, N., Aragó, M., Gámez, A., Esteban, M., Díaz-Cruz, J. M., \& Núñez, O. (2018). Determination of HPLC-UV fingerprints of Spanish paprika (Capsicum annuи L.) for its classification by linear discriminant analysis. Sensors, 18(12), 4479.

Cetó, X., Voelcker, N. H., \& Prieto-Simón, B. (2016). Bioelectronic tongues: New trends and applications in water and food analysis. Biosensors and Bioelectronics, 79, 608-626. doi: http://dx.doi.org/10.1016/j.bios.2015.12.075

Publication of an application for registration pursuant to Article 6(2) of Regulation (EEC) No 2081/92 on the protection of geographical indications and designations of origin (11 February 2000).

Publication of an application pursuant to Article 6(2) of Council Regulation (EC) No $510 / 2006$ on the protection of geographical indications and designations of origin for agricultural products and foodstuffs (24 November 2006).

Cuadros-Rodríguez, L., Ruiz-Samblás, C., Valverde-Som, L., Pérez-Castaño, E., \& González-Casado, A. (2016). Chromatographic fingerprinting: An innovative approach for food 'identitation' and food authentication - A tutorial. Analytica Chimica Acta, 909, 9-23. doi: https://doi.org/10.1016/j.aca.2015.12.042

Danezis, G. P., Tsagkaris, A. S., Camin, F., Brusic, V., \& Georgiou, C. A. (2016). Food authentication: Techniques, trends \& emerging approaches. TrAC Trends in Analytical Chemistry, 85, 123-132. doi: https://doi.org/10.1016/j.trac.2016.02.026 
330 Dias, C., \& Mendes, L. (2018). Protected designation of origin (PDO), Protected geographical indication (PGI) and traditional speciality guaranteed (TSG): A bibiliometric analysis. Food Research International, 103, 492-508. doi: https://doi.org/10.1016/j.foodres.2017.09.059

Esteki, M., Shahsavari, Z., \& Simal-Gandara, J. (2019). Food identification by high performance liquid chromatography fingerprinting and mathematical processing. Food Research International, 303-317. doi: https://doi.org/10.1016/j.foodres.2019.04.025

Galvin-King, P., Haughey, S. A., \& Elliott, C. T. (2018). Herb and spice fraud; the drivers, challenges and detection. Food Control, 88, 85-97. doi: https://doi.org/10.1016/j.foodcont.2017.12.031

Johanningsmeier, S. D., Harris, G. K., \& Klevorn, C. M. (2016). Metabolomic technologies for improving the quality of food: Practice and promise. Annual Review of Food Science and Technology, 7(1), 413-438. doi: 10.1146/annurev-food022814-015721

Johnson, R. A., \& Wichein, D. W. (2007). Applied multivariate statistical analysis (Vol. 6th). Harlow, GB: Pearson Education.

Kim, H.-G., Bae, J.-H., Jastrzebski, Z., Cherkas, A., Heo, B.-G., Gorinstein, S., \& Ku, Y.-G. (2016). Binding, Antioxidant and anti-proliferative properties of bioactive compounds of sweet paprika (Capsicum annuum L.). Plant Foods for Human Nutrition, 71(2), 129-136. doi: 10.1007/s11130-016-0550-9

Kris-Etherton, P. M., Hecker, K. D., Bonanome, A., Coval, S. M., Binkoski, A. E., Hilpert, K. F., . . Etherton, T. D. (2002). Bioactive compounds in foods: their role in the prevention of cardiovascular disease and cancer. The American Journal of Medicine, 113(9), 71-88. doi: 10.1016/S0002-9343(01)00995-0

Mudrić, S. Ž., Gašić, U. M., Dramićanin, A. M., Ćirić, I. Ž., Milojković-Opsenica, D. M., Popović-Đorđević, J. B., . . . Tešić, Ž. L. (2017). The polyphenolics and carbohydrates as indicators of botanical and geographical origin of Serbian autochthonous clones of red spice paprika. Food Chemistry, 217, 705-715. doi: https://doi.org/10.1016/j.foodchem.2016.09.038

Pérez-Gálvez, A., Jarén-Galán, M., Mínguez-Mosquera, M.I. (2005), Processing of red pepper fruits (Capsicum Annuum L.) for production of paprika and paprika oleoresin. Handbook of Fruits and Fruit Processing, Y. H. Hui Ed. (pp. 565-579). 
363 Quideau, S., Deffieux, D., Douat-Casassus, C., \& Pouységu, L. (2011). Plant 364 polyphenols: Chemical properties, biological activities, and synthesis. Angewandte 365 Chemie International Edition, 50(3), 586-621. doi: 10.1002/anie.201000044 366 Zeng, Z., Chau, F.-t., Chan, H.-y., Cheung, C.-y., Lau, T.-y., Wei, S., . . . Liang, Y. 367 (2008). Recent advances in the compound-oriented and pattern-oriented approaches 368 to the quality control of herbal medicines. Chinese Medicine, 3(1), 9. doi: $369 \quad 10.1186 / 1749-8546-3-9$ 
Table 1. Composition of the set of samples prepared to evaluate paprika adulteration based on its type [(A) sweet, (B) bittersweet and (C) spicy] as well as on its region [(A) La Vera, (B) Murcia and (C) Czech Republic].

\begin{tabular}{cccccccccccc}
\hline Class & $\mathbf{A}$ & $\mathbf{B}$ & $\mathbf{C}$ & Class & $\mathbf{A}$ & $\mathbf{B}$ & $\mathbf{C}$ & Class & $\mathbf{A}$ & $\mathbf{B}$ & $\mathbf{C}$ \\
\hline 1 & 1 & 0 & 0 & 9 & 0 & 1 & 0 & 17 & 0 & 0 & 1 \\
2 & 0.99 & 0.01 & 0 & 10 & 0 & 0.99 & 0.01 & 18 & 0.01 & 0 & 0.99 \\
3 & 0.98 & 0.02 & 0 & 11 & 0 & 0.98 & 0.02 & 19 & 0.02 & 0 & 0.98 \\
4 & 0.9 & 0.1 & 0 & 12 & 0 & 0.9 & 0.1 & 20 & 0.1 & 0 & 0.9 \\
5 & 0.8 & 0.2 & 0 & 13 & 0 & 0.8 & 0.2 & 21 & 0.2 & 0 & 0.8 \\
6 & 0.6 & 0.4 & 0 & 14 & 0 & 0.6 & 0.4 & 22 & 0.4 & 0 & 0.6 \\
7 & 0.4 & 0.60 & 0 & 15 & 0 & 0.4 & 0.60 & 23 & 0.60 & 0 & 0.4 \\
8 & 0.2 & 0.8 & 0 & 16 & 0 & 0.2 & 0.8 & 24 & 0.8 & 0 & 0.2 \\
\hline
\end{tabular}




\section{FIGURE CAPTIONS}

Figure 1. Representative raw chromatograms obtained for spicy paprika samples extracts of (top to bottom) La Vera, Murcia and Czech Republic under the conditions described in section 2.3.

Figure 2. Score plot obtained after LDA analysis for the authentication of paprika's type. In this study 144 chromatograms were analysed, corresponding to 24 different proportions of the sweet, bittersweet and spicy types of paprika from La Vera (two samples for every proportion and three injections per sample). Numbers indicate the class of every sample (i.e., the proportion of paprika types) according to Table 1. Coloured filled symbols correspond to the training subset and black empty ones to the testing subset, whereas the centroid for each of the classes is also plotted $(\star)$.

Figure 3. Performance of the optimized FFT-PLS model for the authentication of paprika's type. For every sample, the predicted versus expected percentage of adulteration of sweet paprika from La Vera with the bittersweet variety of the same PDO is shown, including training $(\bullet$, solid line $)$ and testing $(\odot$, dotted line $)$ subsets. The dashed line corresponds to the theoretical diagonal line.

Figure 4. Score plot obtained after LDA analysis for the authentication of paprika's region. In this study 144 chromatograms were analysed, corresponding to 24 different proportions of the spicy type of paprika from La Vera, Murcia and Czech Republic (two samples for every proportion and three injections per sample). Numbers indicate the class of every sample (i.e., the proportion of paprika types) according to Table 1. 
Coloured filled symbols correspond to the training subset and black empty ones to the testing subset, whereas the centroid for each of the classes is also plotted ( $\star$ ).

Figure 5. Performance of the optimized FFT-PLS model for the authentication of paprika's region. For every sample, the predicted versus expected percentage of adulteration of spicy paprika from La Vera with the same variety from Murcia is shown, including training $(\bullet$, solid line) and testing $(\odot$, dotted line) subsets. The dashed line corresponds to the theoretical diagonal line. 
Fig1

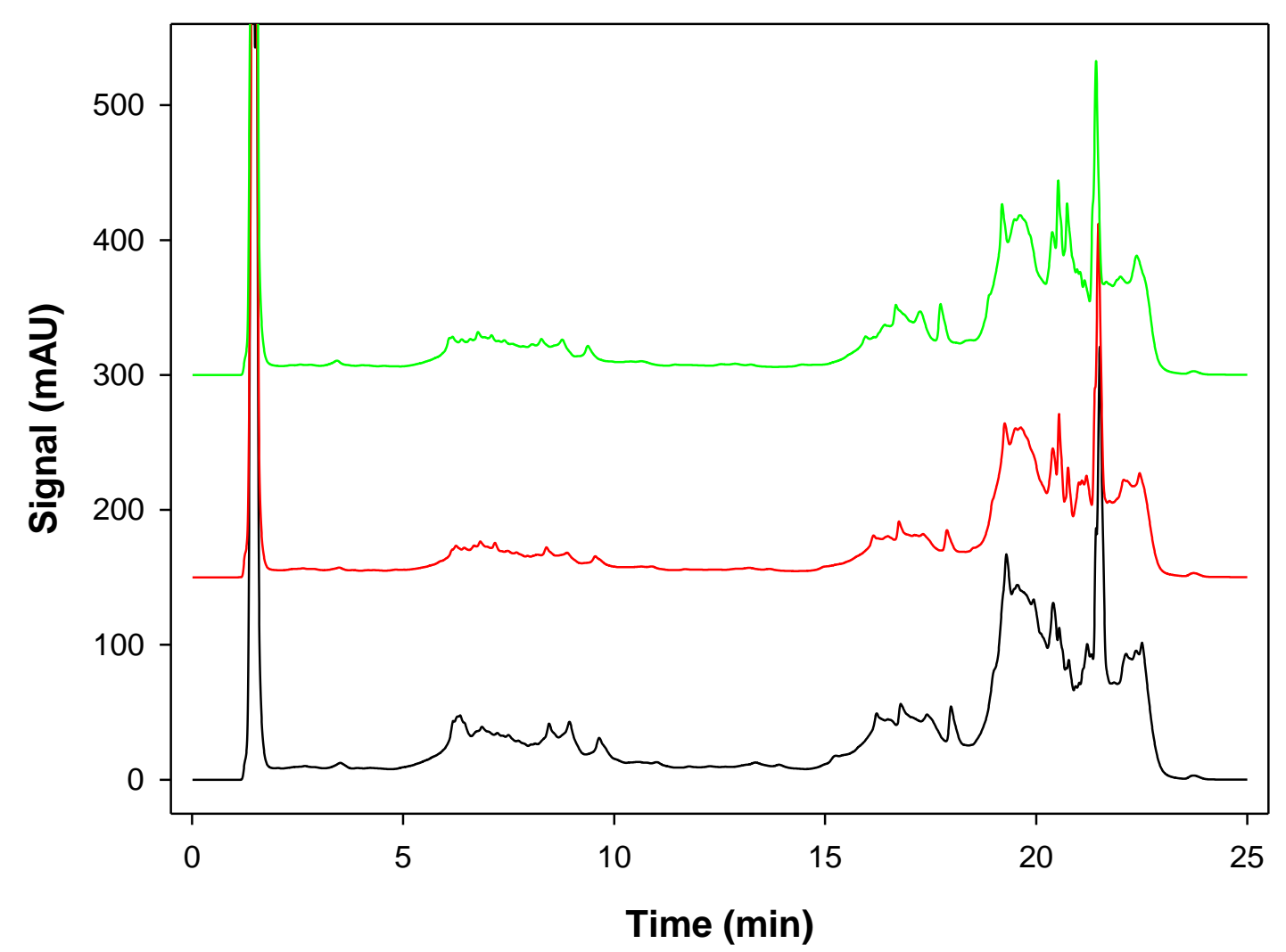


Fig3

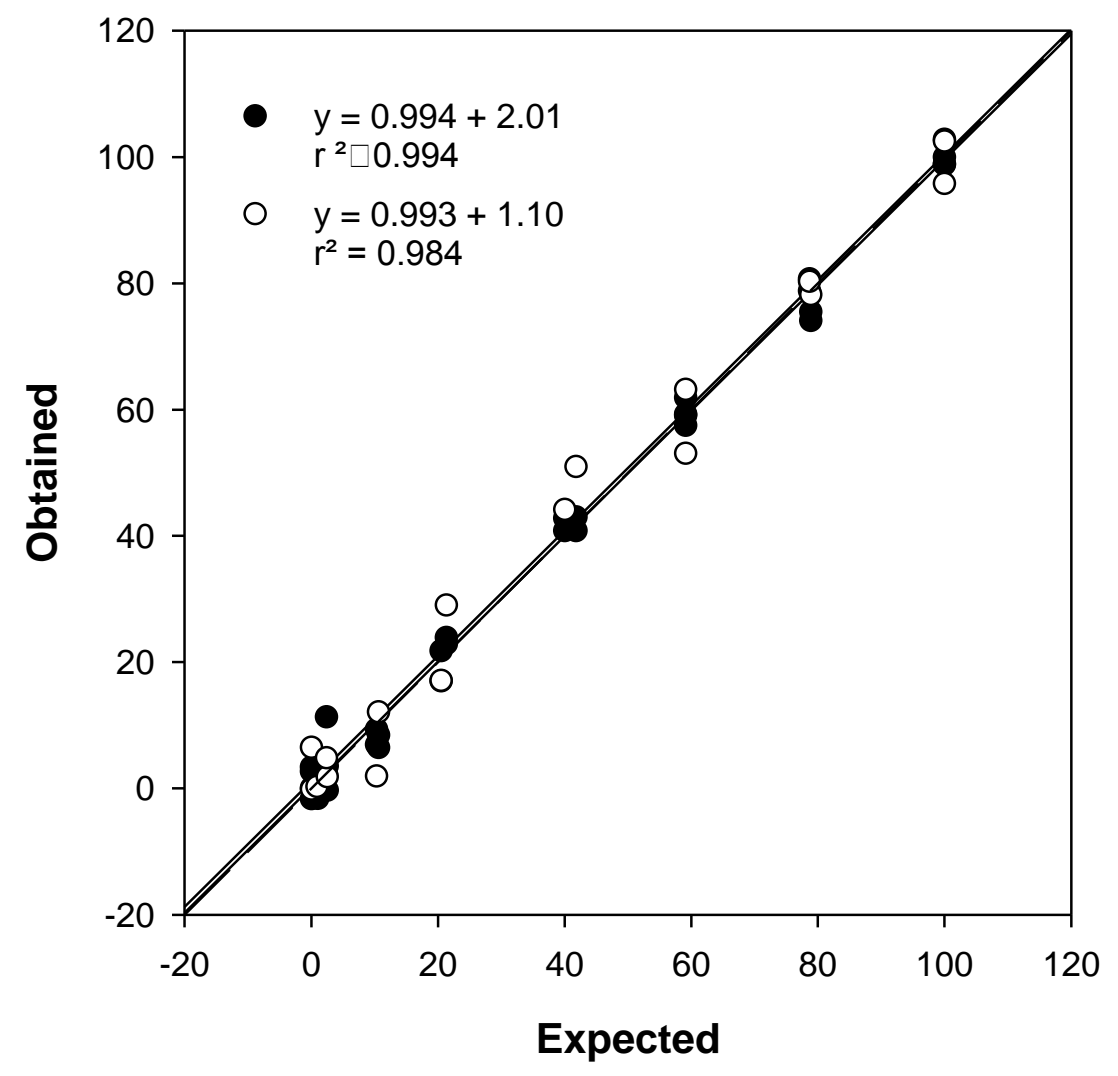


Fig4

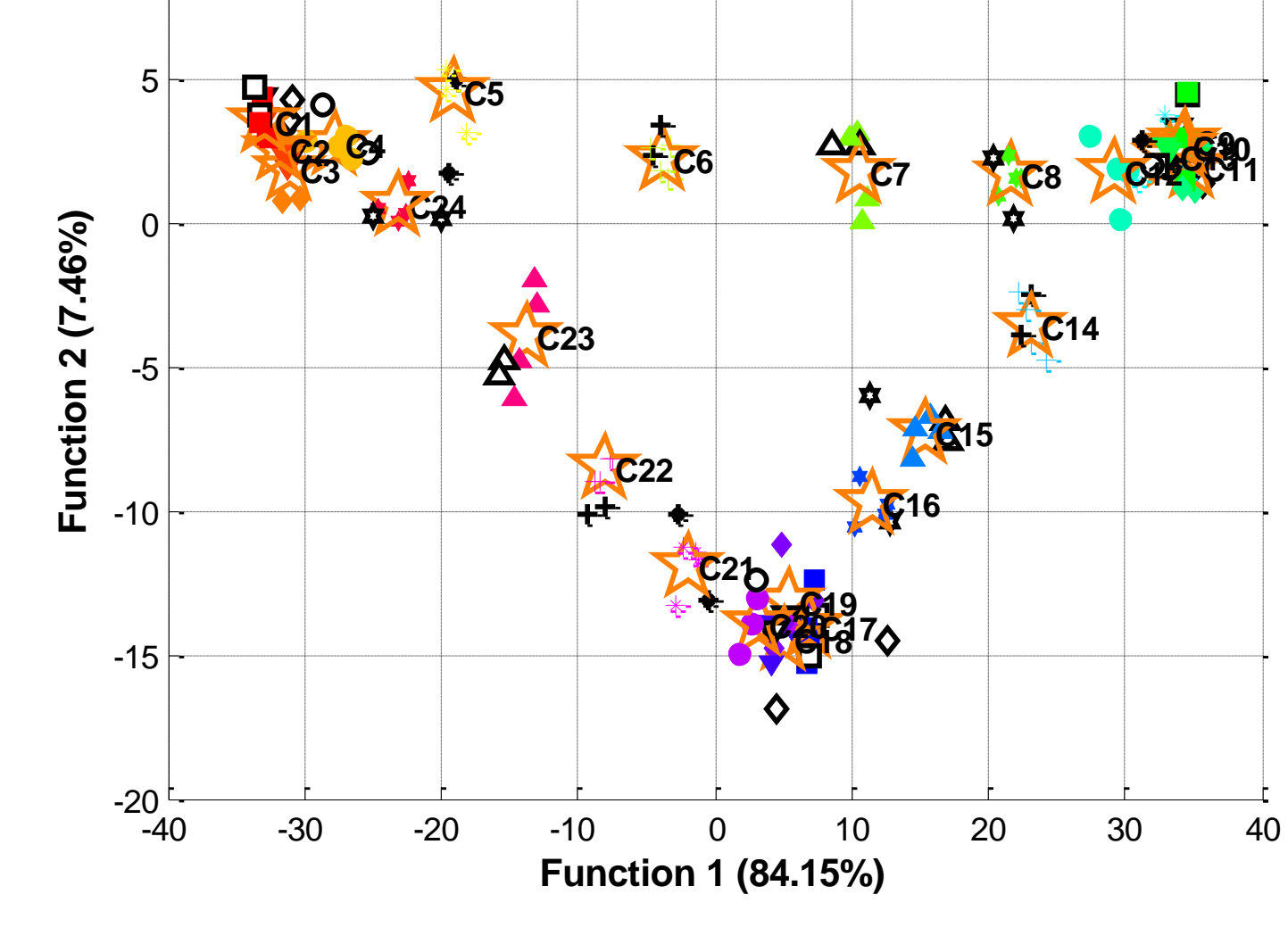


Fig5

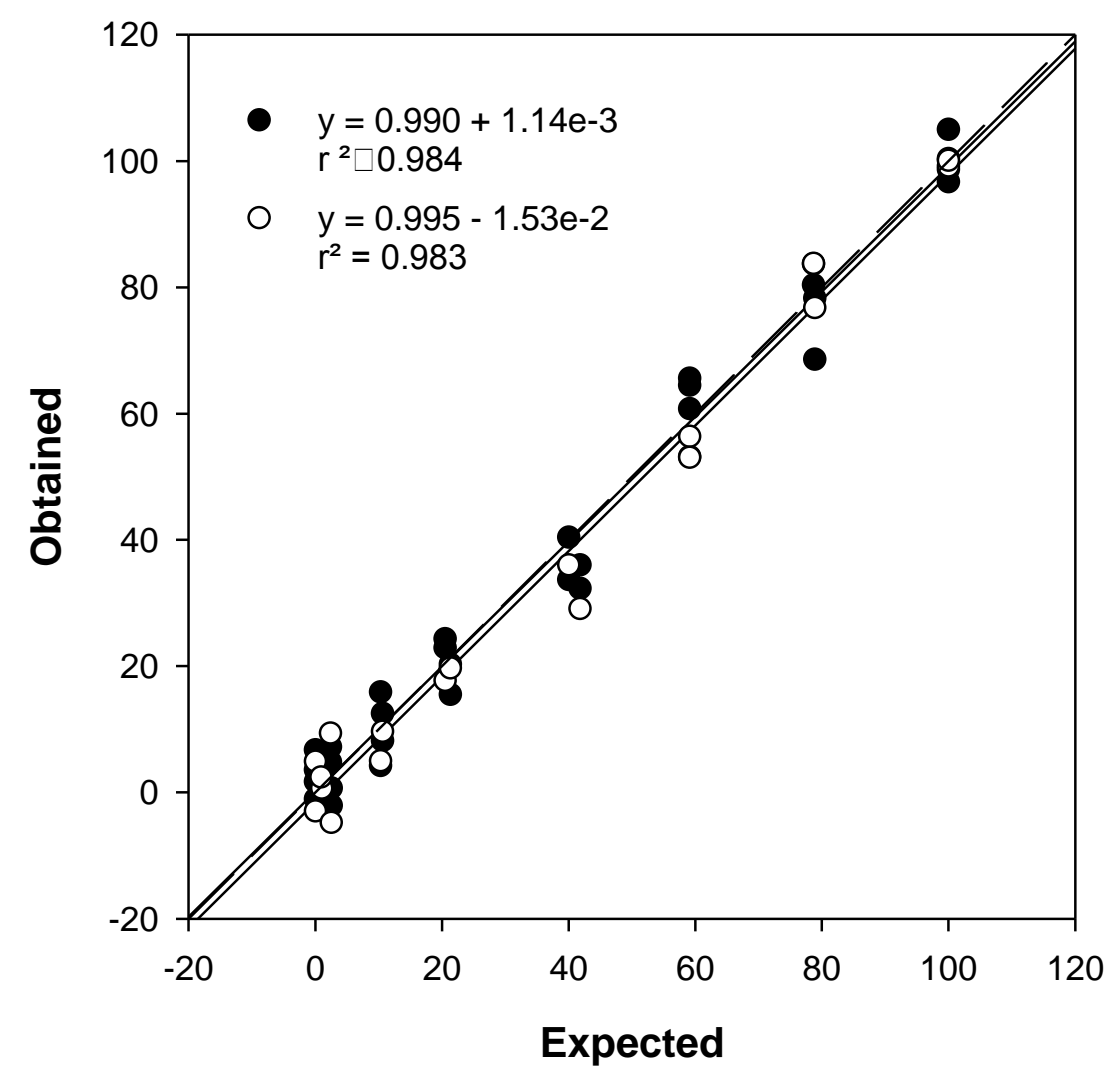




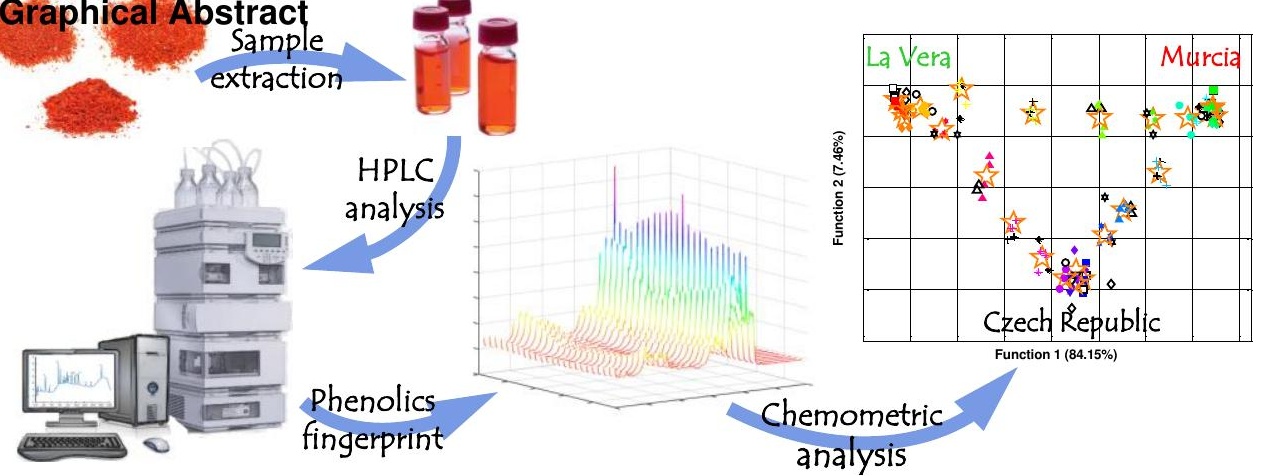


${ }^{*}$ Conflict of Interest Form

\section{Conflicts of interest}

Declarations of interest: none 


\section{Authors contribution section}

This work was originally designed by Núria Serrano, Oscar Núñez and José Manuel Díaz-Cruz. Cristina Sánchez carried out the experimental work by liquid chromatography. Xavier Cetó contributed to the design of experiments and carried out the data treatment. He also wrote the initial version of the manuscript that was later improved with contributions by Núria Serrano, Oscar Núñez and José Manuel DíazCruz. 